Article

\title{
On Approximation of Any Ordered Fuzzy Number by A Trapezoidal Ordered Fuzzy Number
}

\author{
Krzysztof Piasecki ${ }^{1, *(\mathbb{D})}$ and Anna Łyczkowska-Hanćkowiak ${ }^{2}$ (]) \\ 1 Department of Investment and Real Estate, Poznan University of Economics and Business, al. \\ Niepodleglosci 10, 61-875 Poznań, Poland \\ 2 Department of Finance, WSB University in Poznań, ul. Powstańców Wielkopolskich 5, \\ 61-895 Poznań, Poland; anna.lyczkowska-hanckowiak@wsb.poznan.pl \\ * Correspondence: krzysztof.piasecki@ue.poznan.pl; Tel.: +48-61-854-3531
}

Received: 5 September 2018; Accepted: 18 October 2018; Published: 21 October 2018

\begin{abstract}
In this paper, the model of imprecise quantity information is an ordered fuzzy number. The purpose of our study is to propose some methods of approximating any ordered fuzzy number using a trapezoidal ordered fuzzy number. The information ambiguity is evaluated by means of an energy measure. The information indistinctness is evaluated by Kosko's entropy measure. We discuss the problem of approximation of an arbitrary ordered fuzzy number by the nearest trapezoidal ordered fuzzy number. This way, we can simplify arithmetical operations on the linear space of ordered fuzzy numbers. The set of feasible trapezoidal ordered numbers is limited by the combination of the following conditions: invariance of energy measure, invariance of entropy measure, and invariance of information support. Evaluating the influence of individual limits combinations on the utility of given approximations, two combinations of those restraints, recommended for use, were chosen. It was also indicated that one of the recommended approximation problems can be used only for ordered fuzzy numbers characterized by a low level of entropy. The obtained results are currently used in such multi-criterial decision making models as financial portfolio management, evaluation of negotiations offers, the fuzzy TOPSIS model, and the fuzzy SAW model.
\end{abstract}

Keywords: ordered fuzzy numbers; energy measure; entropy measure; information ambiguity; information indistinctness

\section{Introduction}

Ordered fuzzy numbers (OFN) are defined in the perfect intuitive way by Kosiński et al. [1-4] as an extension of the concept of fuzzy number (FN). In this way, they were going to introduce a FN supplemented by a negative or positive orientation. The negative orientation means the order from bigger numbers to smaller ones. The positive orientation means the order from smaller numbers to bigger ones. The FN orientation is interpreted as a prediction of future FN changes. The Kosiński' theory has significant drawbacks. Kosiński [4] has shown that there exist improper OFNs that cannot be represented $b$. This means that we cannot apply any knowledge of fuzzy sets to solve practical problems described by improper OFN. Therefore, considerations that use improper OFN may not be fruitful. Kosiński [1-3] has determined OFNs' arithmetic as an extension of results obtained by Goetschel and Voxman [5] for FNs. Moreover, Kosiński [4] has shown that there exist such proper OFNs that their Kosiński's sum is equal to improper OFN. This means that the family of all proper OFNs is not closed under the Kosiński's addition. On the other hand, most mathematical applications require that the considered objects' family be closed under used arithmetic operations. Then again, 
the intuitive Kosiński's approach to the notion of OFN is very useful. For this reason, the original Kosiński's theory was revised in [6]. This paper is fully based on the revised OFNs' theory.

OFNs have already begun to find their use in operations research applied in decision making, economics, and finance [7-31]. All discussed applications are associated with the linear space of OFNs. However, a big inconvenience appears when using that space. The addition of an arbitrary OFN is a very complicated operation of significant formal complexity. An example of this addition is presented in Section 2. On the other hand, the addition of trapezoidal OFNs (TrOFN) is reduced to a simple addition of four-dimensional vectors of real numbers. Moreover, TrOFN are already applied for finance $[20-22,25]$ and decision making $[14,26,29,30]$. These are the main premises to propose a substitution of OFNs by TrOFNs. It is obvious that any arbitrary OFN should be substituted by the nearest TrOFN. Therefore, we will apply the approximation task as a tool of substitution of OFN by TrOFN. When processing imprecise values, we use OFN only to follow the influence of the initial values' imprecision on the resulting information imprecision. Due to our approximation problem, we can impose the requirements of approximating by such a value that retains the estimates of the imprecision of an approximated value. The approximation problem of OFNs by TrOFNs has not, so far, been discussed in the literature [32].

The main aim of this paper is to propose the approximation methods of any OFN by trapezoidal OFNs. As a result, authors intend to indicate the recommended approximation methods. The paper is organised as follows. Section 2 presents the concept of OFNs and the linear space of OFNs. Section 3 briefly discusses the idea of imprecision [33]. The same chapter describes energy measure [34] as a measure tool of information ambiguity and entropy measure [35,36], as well as a measure tool of information indistinctness. In Section 4, the authors propose six approximation methods of any OFN by a TrOFN. Section 5 recommends chosen approximation methods and shows the possibility of implementing obtained results in a case of existing models of real objects. Finally, Section 6 concludes the article, summarizes the main findings of this research, and proposes some future research directions.

\section{Ordered Fuzzy Numbers-Basic Facts}

By $\mathcal{F}(\mathbb{R})$, we denote the family of all fuzzy subsets of a real line $\mathbb{R}$. An imprecise number is a family of values in which each considered value belongs to it in a varying degree. A commonly accepted model of an imprecise number is the fuzzy number (FN), defined as a fuzzy subset of the real line $\mathbb{R}$. The most general definition of FN was given by Dubois and Prade [37]. Thanks to the results obtained in [5], a concept of a fuzzy number can be equivalently denoted as follows:

Theorem 1. For any $F N \mathcal{L}$ there exists such a nondecreasing sequence $(a, b, c, d) \subset \mathbb{R}$ that $\mathcal{L} \in \mathcal{F}(\mathbb{R})$ is represented by its membership function $\mu_{\mathcal{L}}\left(\cdot \mid a, b, c, d, L_{L}, R_{L}\right) \in[0 ; 1]^{\mathbb{R}}$ given by the identity

$$
\mu_{\mathcal{L}}\left(x \mid a, b, c, d, L_{L}, R_{L}\right)= \begin{cases}0, & x \notin[a, d], \\ L_{L}(x), & x \in[a, b], \\ 1, & x \in[b, c], \\ R_{L}(x), & x \in[c, d],\end{cases}
$$

in which the left reference function $L_{L} \in[0 ; 1]^{[a, b]}$ and the right reference function $R_{L} \in[0 ; 1]^{[c, d]}$ are upper semi-continuous monotonic functions satisfying the following conditions:

$$
\begin{gathered}
L_{L}(b)=R_{L}(c)=1, \\
\forall_{x \in] a, d[}: \mu_{\mathcal{L}}\left(x \mid a, b, c, d, L_{L}, R_{L}\right)>0 .
\end{gathered}
$$

The family of all FN is denoted as $\mathbb{F}$. Moreover, Dubois and Prade [38] have introduced such arithmetic operations on FN that are coherent with the Zadeh Extension Principle. 
The concept of ordered fuzzy numbers (OFN) was intuitively introduced by Kosiński and his co-writers in the series of papers [1-4] as an extension of the concept of FN. A significant drawback of Kosiński's theory is that there exist such OFNs that, in fact, are not FN [4]. What is more, the intuitive Kosiński's approach to the notion of OFN is very useful. The OFNs' usefulness results from the fact that an OFN is defined as FN supplemented by a negative or positive orientation. The negative orientation means the order from bigger numbers to smaller ones. The positive orientation means the order from smaller numbers to bigger ones. The FN orientation is interpreted as prediction of future FN changes. For this reason, the Kosiński's theory was revised in [6], in which OFNs are generally defined in a following way:

Definition 1. Let any monotonic sequence $(a, b, c, d) \subset \mathbb{R}$ of ordered fuzzy number $(\mathrm{OFN}) \stackrel{\leftrightarrow}{\mathcal{L}}\left(a, b, c, d, S_{L}, E_{L}\right)$ be defined as the pair of fuzzy number $\mathcal{L} \in \mathbb{F}$ and orientation $a \mapsto d=(a, d)$. The fuzzy number $\mathcal{L} \in \mathbb{F}$ is determined by its membership function $\mu_{\mathcal{L}}\left(\cdot \mid a, b, c, d, S_{L}, E_{L}\right) \in[0 ; 1]^{\mathbb{R}}$ given by the identity

$$
\mu_{\mathcal{L}}\left(x \mid a, b, c, d, S_{L}, E_{L}\right)= \begin{cases}0, & x \notin[a, d]=[d, a], \\ S_{L}(x), & x \in[a, b]=[b, a], \\ 1, & x \in[b, c]=[c, b], \\ E_{L}(x), & x \in[c, d]=[d, c]\end{cases}
$$

in which the starting-function $S_{L} \in[0 ; 1]^{[a, b]}$ and the ending-function $E_{L} \in[0 ; 1]^{[c, d]}$ are upper semi-continuous monotonic functions satisfying the conditions

$$
\begin{gathered}
S_{L}(b)=E_{L}(c)=1 . \\
\forall_{x \in] a, d[} \mu_{\mathcal{L}}\left(x \mid a, b, c, d, S_{L}, E_{L}\right)>0 .
\end{gathered}
$$

Remark: Let us note that Equation (4) identity describes additionally extended notation of numerical intervals, which is used in this work.

The space of all OFN is denoted by the symbol $\mathbb{K}$. The condition $a<d$ fulfilment determines the positive orientation $a \longmapsto d$ of OFN $\overleftrightarrow{\mathcal{L}}\left(a, b, c, d, S_{L}, E_{L}\right)$. In this case, the starting-function $S_{L}$ is non-decreasing and the ending-function $E_{L}$ is non-increasing. Any positively oriented OFN is interpreted as imprecise number, which may increase. The space of all positively oriented OFN is denoted by the symbol $\mathbb{K}^{+}$. The condition $a>d$ fulfilment determines the negative orientation of OFN $\overleftrightarrow{\mathcal{L}}\left(a, b, c, d, S_{L}, E_{L}\right)$. In this case, the starting-function $S_{L}$ is non-increasing and the ending-function $E_{L}$ is non-decreasing. Negatively oriented OFN is interpreted as an imprecise number, which may decrease. The space of all negatively oriented OFN is denoted by the symbol $\mathbb{K}^{-}$. For the case $a=d$, OFN $\overleftrightarrow{\mathcal{L}}\left(a, a, a, a, S_{L}, E_{L}\right)$ represents a crisp number $a \in \mathbb{R}$, which is not oriented. In summary, we can write

$$
\mathbb{K}=\mathbb{K}^{+} \cup \mathbb{R} \cup \mathbb{K}^{-}
$$

For the case $a \geq d$, any FN $\mathcal{L}\left(a, b, c, d, S_{L}, E_{L}\right)$ is equal to OFN $\stackrel{\leftrightarrow}{\mathcal{L}}\left(a, b, c, d, S_{L}, E_{L}\right)$. On the other hand, for this case any OFN $\overleftrightarrow{\mathcal{L}}\left(a, b, c, d, S_{L}, E_{L}\right)$ is equal to FN $\mathcal{L}\left(a, b, c, d, S_{L}, E_{L}\right)$. These facts imply that we have

$$
\begin{aligned}
& \mathbb{F}=\mathbb{K}^{+} \cup \mathbb{R}, \\
& \mathbb{K}=\mathbb{F} \cup \mathbb{K}^{-} .
\end{aligned}
$$

Also, the arithmetic proposed by Kosiński has a significant disadvantage. The space of ordered fuzzy numbers is not closed under Kosiński's addition. Therefore, Kosinski's theory is modified in this way so that the space of ordered fuzzy numbers is closed under revised arithmetic operations. The necessary arithmetic operators will generally be defined for OFNs using the following concepts. 
Definition 2. Cut-function $L^{\star} \in[u, v]^{[0 ; 1]}$ of any upper semi-continuous nondecreasing function $L \in[0 ; 1]^{[u ; v]}$ is given by the identity

$$
L^{\star}(\alpha)=\min \{x \in[u, v]: L(x) \geq \alpha\} .
$$

Definition 3. Cut-function $R^{\star} \in[u, v]^{[0 ; 1]}$ of any upper semi-continuous nonincreasing function $R \in[0 ; 1]^{[u ; v]}$ is given by the identity

$$
R^{\star}(\alpha)=\max \{x \in[u, v]: R(x) \geq \alpha\} .
$$

Definition 4. Pseudo inverse function $l^{\triangleleft} \in[0 ; 1]^{[l(0), l(1)]}$ of any bounded continuous and nondecreasing function $l \in[l(0), l(1)]^{[0 ; 1]}$ is given by the identity

$$
l^{\triangleleft}(x)=\max \{\alpha \in[0 ; 1]: l(\alpha)=x\} .
$$

Definition 5. Pseudo inverse function $r^{\triangleleft} \in[0 ; 1]^{[r(1), r(0)]}$ of any bounded continuous and nonincreasing function $r \in[r(0), r(1)]^{[0 ; 1]}$ is given by the identity

$$
r^{\triangleleft}(x)=\min \{\alpha \in[0 ; 1]: r(\alpha)=x\} .
$$

The vast majority of practical implementations of OFNs is associated with the linear space $\langle\mathbb{K},+, \odot\rangle$, in which

- the symbol + denotes such addition operator on $\mathbb{K}$, which is an extension of the addition operator " +" on $\mathbb{R}$;

- the symbol $\odot$ denotes such dot product operator on $\mathbb{K}$, which is an extension of the dot product operator "." on $\mathbb{R}$.

Therefore, the following considerations will be limited to the case of linear space $\langle\mathbb{K},++\odot\rangle$. Let us consider any pair of OFNs $\overleftrightarrow{\mathcal{K}}, \overleftrightarrow{\mathcal{M}} \in \mathbb{K}$ described as follows

$$
\overleftrightarrow{\mathcal{K}}=\overleftrightarrow{\mathcal{L}}\left(a_{K}, b_{K}, c_{K}, d_{K}, s_{K}, E_{K}\right), \overleftrightarrow{\mathcal{M}}=\overleftrightarrow{\mathcal{L}}\left(a_{M}, b_{M}, c_{M}, d_{M}, s_{M}, E_{M}\right)
$$

and the convention $\frac{0}{0}=1$ is used. For any $\beta \in \mathbb{R}$, the dot product operation on $\mathbb{K}$ is defined by the identity

$$
\beta \odot \overleftrightarrow{\mathcal{M}}=\stackrel{\leftrightarrow}{\mathcal{J}}=\overleftrightarrow{\mathcal{L}}\left(\beta \cdot a_{M}, \beta \cdot b_{M}, \beta \cdot c_{M}, \beta \cdot d_{M}, S_{J}, E_{J}\right)
$$

in which we have

$$
\begin{aligned}
& S_{J}(x)=S_{M}(x / \beta), \\
& E_{J}(x)=S_{M}(x / \beta) .
\end{aligned}
$$

Example 1. Let us take into account the OFN $\overleftrightarrow{\mathcal{U}}=\overleftrightarrow{\mathcal{L}}\left(2,4,8,10, S_{U}, E_{U}\right)$, in which

$$
\mu_{U}(x)=\left\{\begin{array}{cc}
0, & x \notin[2,10], \\
S_{U}(x), & x \in[2,4], \\
1, & x \in[4,8], \\
E_{U}(x), & x \in[8,10],
\end{array}\right\}= \begin{cases}0, & x \notin[2,10], \\
\frac{2 x-4}{x}, & x \in[2,4], \\
1, & x \in[4,8], \\
\frac{3 x-30}{x-14}, & x \in[8,10] .\end{cases}
$$


Then, for example, we have

$$
\overleftrightarrow{\mathcal{W}}=3 \odot \overleftrightarrow{\mathcal{U}}=\overleftrightarrow{\mathcal{L}}\left(3 \cdot 2,3 \cdot 4,3 \cdot 8,3 \cdot 10, S_{W}, E_{W}\right)=\overleftrightarrow{\mathcal{L}}\left(6,12,24,30, S_{W}, E_{W}\right)
$$

in which the conditions (16) and (17) imply

$$
\begin{gathered}
\mu_{W}(x)=\left\{\begin{array}{cc}
0, & x \notin[6,30], \\
S_{W}(x), & x \in[6,12], \\
1, & x \in[12,24], \\
E_{W}(x), & x \in[24,30],
\end{array}\right\}=\left\{\begin{array}{cc}
0, & x \notin[6,30], \\
S_{U}\left(\frac{x}{3}\right), & x \in[6,12], \\
1, & x \in[12,24], \\
E_{U}\left(\frac{x}{3}\right), & x \in[24,30],
\end{array}\right\}= \\
\left\{\begin{array}{cc}
0, & x \notin[6,30], \\
\frac{2 \cdot x-12}{x}, & x \in[6,12], \\
1, & x \in[12,24], \\
\frac{3 \cdot x-90}{x-42}, & x \in[24,30] .
\end{array}\right.
\end{gathered}
$$

The addition operation on $\mathbb{K}$ is defined by the identity [6]

$$
\overleftrightarrow{\mathcal{K}}+\overleftrightarrow{\mathcal{M}}=\overleftrightarrow{\mathcal{J}}=\overleftrightarrow{\mathcal{L}}\left(a_{J}, b_{J}, c_{J}, d_{J}, S_{J}, E_{J}\right)
$$

in which we have

$$
\begin{aligned}
& \check{a}_{J}=a_{K}+a_{M}, \\
& b_{J}=b_{K}+b_{M} \text {, } \\
& c_{J}=c_{K}+c_{M}, \\
& \check{d}_{J}=d_{K}+d_{M} \text {, } \\
& a_{J}= \begin{cases}\min \left\{\check{a}_{J}, b_{J}\right\}, & \left(b_{J}<c_{J}\right) \vee\left(b_{J}=c_{J} \wedge \check{a}_{J} \leq \check{d}_{J}\right), \\
\max \left\{\check{a}_{J}, b_{J}\right\}, & \left(b_{J}>c_{J}\right) \vee\left(b_{J}=c_{J} \wedge \check{a}_{J}>\check{d}_{J}\right),\end{cases} \\
& d_{J}= \begin{cases}\max \left\{\check{d}_{J}, c_{J}\right\}, & \left(b_{J}<c_{J}\right) \vee\left(b_{J}=c_{J} \wedge \check{a}_{J} \leq \check{d}_{J}\right), \\
\min \left\{\check{d}_{J}, c_{J}\right\}, \quad\left(b_{J}>c_{J}\right) \vee\left(b_{J}=c_{J} \wedge \check{a}_{J}>\check{d}_{J}\right),\end{cases} \\
& \forall_{\alpha \in[0 ; 1]} \quad s_{J}(\alpha)=\left\{\begin{array}{cc}
S_{K}^{\star}(\alpha)+S_{M}^{\star}(\alpha), & a_{J} \neq b_{J}, \\
b_{J}, & a_{J}=b_{J},
\end{array}\right. \\
& \forall_{\alpha \in[0 ; 1]} \quad e_{J}(\alpha)=\left\{\begin{array}{c}
E_{K}^{\star}(\alpha)+E_{M}^{\star}(\alpha), \quad c_{J} \neq d_{J}, \\
c_{J}, \quad c_{J}=d_{J},
\end{array}\right. \\
& \forall_{x \in\left[a_{J}, b_{J}\right]} \quad S_{J}(x)=s_{J}^{\triangleleft}(x), \\
& \forall_{x \in\left[c_{J}, d_{J}\right]} \quad E_{J}(x)=e_{J}^{\triangleleft}(x)
\end{aligned}
$$

The above described procedure of OFN's addition is widely justified in [6].

Example 2. Let us take into account the OFNs $\overleftrightarrow{\mathcal{U}}$ given by (18) and $\overleftrightarrow{\mathcal{V}}=\overleftrightarrow{\mathcal{L}}\left(15,11,6,5, S_{V}, E_{V}\right)$, in which

$$
\mu_{V}(x)=\left\{\begin{array}{cc}
0, & x \notin[15,5], \\
S_{V}(x), & x \in[15,11], \\
1, & x \in[11,6], \\
E_{V}(x), & x \in[6,5],
\end{array}\right\}= \begin{cases}0, & x \notin[15,5], \\
\frac{3 x-45}{x-23}, & x \in[15,11], \\
1, & x \in[11,6], \\
\frac{3 x-15}{x-3}, & x \in[6,5] .\end{cases}
$$


We determine the sum

$$
\overleftrightarrow{\mathcal{Z}}=\overleftrightarrow{\mathcal{U}}++\overleftrightarrow{\mathcal{V}}=\overleftrightarrow{\mathcal{L}}\left(a_{Z}, b_{Z}, c_{Z}, d_{Z}, S_{Z}, E_{Z}\right)
$$

From relations (22)-(25) we obtain

$$
\begin{gathered}
\check{a}_{Z}=a_{U}+a_{V}=2+15=17, b_{Z}=b_{U}+b_{V}=4+11=15, \\
c_{Z}=c_{U}+c_{V}=8+6=14, \breve{d}_{Z}=d_{U}+d_{V}=10+5=15 .
\end{gathered}
$$

Because we have $15=b_{Z}>c_{z}=14$, from (26) we get $a_{Z}=17$, and from (27) we get $d_{Z}=14$. Consequently, we get

$$
\overleftrightarrow{\mathcal{Z}}=\overleftrightarrow{\mathcal{U}}+\overleftrightarrow{\mathcal{V}}=\overleftrightarrow{\mathcal{L}}\left(17,15,14,14, S_{Z}, E_{Z}\right)
$$

In the next step, we determine the variability of the ending-function $E_{Z} \in[0 ; 1]^{[14,14]}$. Due to satisfaction of the condition $c_{Z}=14=d_{Z}$, the Equation (29) implies

$$
\forall_{\alpha \in[0 ; 1]} \quad e_{Z}(\alpha)=c_{Z}=14
$$

Then, from the relations (13) and (31), we obtain

$$
\forall_{x \in[14,14]} E_{Z}(x)=E_{Z}(14)=e_{M}^{\triangleleft}(14)=\max \left\{\alpha \in[0 ; 1]: e_{Z}(\alpha)=14\right\}=1 .
$$

Next, we establish the variability of the starting-function $S_{Z} \in[0 ; 1]^{[17,15]}$. Here, we have $a_{Z}=17 \neq$ $15=b_{Z}$. Starting-function $S_{U} \in[0 ; 1]^{[2,4]}$ is a non-decreasing one. It is very easy to check that this function is increasing. Therefore, from (10) for any $\alpha \in[0 ; 1]$ we have

$$
S_{U}^{\star}(\alpha)=\min \left\{x \in[2,4]: S_{U}(x) \geq \alpha\right\}=S_{U}^{-1}(\alpha)=\frac{4}{2-\alpha} .
$$

Starting-function $S_{V} \in[0 ; 1]^{[15,11]}$ is a non-increasing function. It is very easy to check that this function is decreasing. Therefore, from (10) for any $\alpha \in[0 ; 1]$ we have

$$
S_{V}^{\star}(\alpha)=\max \left\{x \in[15,11]: S_{V}(x) \geq \alpha\right\}=S_{V}^{-1}(\alpha)=\frac{23 \alpha-45}{\alpha-3} .
$$

Next, from (28) we obtain

$$
s_{Z}(\alpha)=S_{U}^{\star}(\alpha)+S_{V}^{\star}(\alpha)=\frac{4}{2-\alpha}+\frac{23 \alpha-45}{\alpha-3}=\frac{23 \alpha^{2}-95 \alpha+102}{\alpha^{2}-5 \alpha+6} .
$$

It is very easy to check whether the function $s_{Z} \in[17,15]^{[0,1]}$ is decreasing. Therefore, from the identities (13) and (30), we obtain

$$
\begin{aligned}
& S_{Z}(x)=s_{Z}^{\triangleleft}(x)=\min \left\{\alpha \in[0 ; 1]: s_{Z}(\alpha)=x\right\}=\min \left\{\alpha \in[0 ; 1]: \frac{23 \alpha^{2}-95 \alpha+102}{\alpha^{2}-5 \alpha+6}=x\right\} \\
& \quad=\min \left\{\frac{5(x-19)-\sqrt{x^{2}+10 x-359}}{2(x-23)}, \frac{5(x-19)+\sqrt{x^{2}+10 x-359}}{2(x-23)}\right\}=\frac{5(x-19)+\sqrt{x^{2}+10 x-359}}{2(x-23)} .
\end{aligned}
$$

Eventually, what we obtain is that the sum $\overleftrightarrow{\mathcal{K}}+\overleftrightarrow{\mathcal{M}}=\overleftrightarrow{\mathcal{Z}}=\overleftrightarrow{\mathcal{L}}\left(17,15,14,14, S_{Z}, E_{Z}\right)$ is unambiguously determined by its membership function

$$
\mu_{Z}(x)=\left\{\begin{array}{cc}
0, & x \notin[17,14], \\
S_{Z}(x), & x \in[17,15], \\
1, & x \in[15,14], \\
E_{Z}(x), & x \in[14,14],
\end{array}\right\}=\left\{\begin{array}{cc}
0, & x \notin[17,4], \\
\frac{5(x-19)+\sqrt{x^{2}+10 x-359},}{2(x-23)} & x \in[17,15], \\
1, & x \in[15,14] \\
1, & x \in[14,14] .
\end{array}\right.
$$


The above example sufficiently shows a high level of formal complexity upon the addition of any OFN. Therefore, in many practical applications researchers limit the use of OFN only to a form presented below.

Definition 2. [6] For any monotonic sequence $(a, b, c, d) \subset \mathbb{R}$ the trapezoidal ordered fuzzy number (TrOFN) $\overleftrightarrow{\operatorname{Tr}}(a, b, c, d)$ is defined as the pair of $F N \mathcal{T} \nabla(a, b, c, d) \in \mathbb{F}$ and the orientation $a \longmapsto d$. The fuzzy number $\mathcal{T} \nabla(a, b, c, d) \in \mathbb{F}$ is determined by its membership function $\mu_{\overparen{T r}}(\cdot \mid a, b, c, d) \in[0 ; 1]^{\mathbb{R}}$ given by the identity

$$
\mu_{\operatorname{Tr}}^{\leftrightarrow}(x \mid a, b, c, d)=\left\{\begin{array}{cc}
0, & x \notin[a, d]=[d, a], \\
\frac{x-a}{b-a}, & x \in[a, b]=] b, a], \\
1, & x \in[b, c]=[c, b], \\
\frac{x-d}{c-d} & x \in] c, d]=[d, c[,
\end{array}\right.
$$

The space of all TrOFN is denoted by the symbol $\mathbb{K}_{T r}$. The following numbers are a particular case of TrOFN.

Definition 3. For any monotonic sequence $(a, b, c) \subset \mathbb{R}$, the triangle ordered fuzzy number $(T O F N) \stackrel{\leftrightarrow}{T}(a, b, c)$ is defined by the identity

$$
\overleftrightarrow{T}(a, b, c)=\overleftrightarrow{\operatorname{Tr}}(a, b, b, c)
$$

For the case of any real number $\beta \in \mathbb{R}$ and any $\operatorname{TrOFN} \overleftrightarrow{\operatorname{Tr}}(a, b, c, d)$, their dot product can be calculated as follows:

$$
\beta \odot \stackrel{\leftrightarrow}{\operatorname{Tr}}(a, b, c, d)=\overleftrightarrow{\operatorname{Tr}}(\beta \cdot a, \beta \cdot b, \beta \cdot c, \beta \cdot d)
$$

Example 3. For example, we have

$$
\overleftrightarrow{\mathcal{W}}=3 \odot \overleftrightarrow{\operatorname{Tr}}(2,4,8,10)=\overleftrightarrow{\operatorname{Tr}}(3 \cdot 2,3 \cdot 4,3 \cdot 8,3 \cdot 10)=\overleftrightarrow{\operatorname{Tr}}(6,12,24,30)
$$

In agreement with arithmetic introduced in [6], for the case of any $\operatorname{TrOFNs} \stackrel{\leftrightarrow}{\operatorname{Tr}}(a, b, c, d)$ and $\overleftrightarrow{\operatorname{Tr}}(p-a, q-b, r-c, s-d)$, their sum is determined as follows:

$$
\begin{gathered}
\stackrel{\leftrightarrow}{\operatorname{Tr}}(a, b, c, d) \boxplus \overleftrightarrow{\operatorname{Tr}}(p-a, q-b, r-c, s-d) \\
= \begin{cases}\stackrel{\leftrightarrow}{\operatorname{Tr}}(\min \{p, q\}, q, r, \max \{r, s\}), & (q<r) \vee(q=r \wedge p \leq s), \\
\stackrel{\leftrightarrow}{\operatorname{Tr}}(\max \{p, q\}, q, r, \min \{r, s\}), & (q>r) \vee(q=r \wedge p>s) .\end{cases}
\end{gathered}
$$

Example 4. We determine the sum

$$
\overleftrightarrow{\mathcal{W}}=\stackrel{\leftrightarrow}{\operatorname{Tr}}(2,4,8,10)++\stackrel{\leftrightarrow}{\operatorname{Tr}}(15,11,6,5)
$$

We have $p=2+15=17, q=4+11=15>14=8+6=r$, and $s=10+5=15$. Then, from (47) we get

$$
\overleftrightarrow{\mathcal{W}}=\overleftrightarrow{\operatorname{Tr}}(17,15,14,14)
$$

Comparing examples 1, 2, 3, and 4 we can easily notice the benefits resulting from using TrOFN when implementing OFN in real object models. The postulate of being constrained to TrOFN use 
when constructing models of real objects is not always possible to satisfy. A behavioural present value $[17,18,39,40]$, described by such OFN that cannot be TrOFN, is an example. The attempts to use this concept in portfolio analysis produce many problems.

All these reservations lead to a postulate to approximate all OFNs by TrOFNs while modelling all real objects. Such an approximation task will be formulated in Section 4 of the paper.

\section{Evaluation of Imprecision}

After [33], we understand imprecision as a superposition of ambiguity and indistinctness of information. Ambiguity can be interpreted as a lack of a clear recommendation between one alternative and various others. Indistinctness is understood as a lack of explicit distinction between recommended and unrecommended alternatives.

Fuzzy subsets [41] are widely used as a model of imprecision information [42,43]. Any OFN is a particular kind of imprecision information. For this reason, in this chapter-if necessary-any OFN $\overleftrightarrow{\mathcal{L}}\left(a, b, c, d, S_{L}, E_{L}\right)$ will be considered as an ordered pair $(\mathcal{L},(a, d)) \in \mathcal{F}(\mathbb{R}) \times \mathbb{R}^{2}$ in which the fuzzy subset $\mathcal{L}$ is determined by its membership function $\mu_{\mathcal{L}} \in[0,1]^{\mathbb{R}}$ given by the identity (4).

An increase in information imprecision reduces the suitability of this information. Therefore, it is logical to consider the problem of imprecision assessment. A basic tool used in this chapter to measure the imprecision of fuzzy sets is Khalili's measure [44] determined by the identity

$$
m(\mathcal{A})=\int_{-\infty}^{+\infty} \mu_{\mathcal{A}}(x) d x
$$

in which the fuzzy subset $\mathcal{A} \in \mathcal{F}(\mathbb{R})$ has bounded support, i.e., its membership function $\mu_{\mathcal{L}} \in[0,1]^{\mathbb{R}}$ fulfils the condition

$$
\exists_{(p, q) \in \mathbb{R}^{2}}\left\{x \in \mathbb{R}: \mu_{\mathcal{A}}(x)>0\right\} \subset[p, q] .
$$

The increase in the ambiguity of an OFN suggests a higher number of alternative recommendations to choose from. This leads to an increase in the risk of choosing an incorrect assessment from recommended alternative ones. This may result in making a decision, which will be ex post associated with a loss of chance. Therefore, an increase in the ambiguity of OFN implies the decrease in the utility of information described by OFN. The proper tool for measuring the ambiguity of FN is an energy measure $d \in\left[\mathbb{R}_{0}^{+}\right]^{\mathcal{F}(\mathbb{R})}$ proposed by de Luca and Termini [35]. In this article, for an arbitrary OFN $\overleftrightarrow{\mathcal{L}}\left(a, b, c, d, S_{L}, E_{L}\right) \in \mathbb{K}$ represented by $\mathcal{L} \in \mathcal{F}(\mathbb{R})$ we have

$$
d\left(\stackrel{\leftrightarrow}{\mathcal{L}}\left(a, b, c, d, S_{L}, E_{L}\right)\right)=m(\mathcal{L})
$$

A new tool for OFN ambiguity evaluation is introduced in [19]. There, it is proposed that the oriented energy index $a \in \mathbb{R}^{\mathbb{K}}$, which assesses the ambiguity of any $\operatorname{OFN} \stackrel{\leftrightarrow}{\mathcal{L}}\left(a, b, c, d, S_{L}, E_{L}\right)$ by integral, was as follows

$$
a\left(\stackrel{\leftrightarrow}{\mathcal{L}}\left(a, b, c, d, S_{L}, E_{L}\right)\right)=\int_{a}^{d} \mu_{\mathcal{L}}(x) d x
$$

in which $\mu_{\mathcal{L}} \in[0,1]^{\mathbb{R}}$ is the membership function of the fuzzy subset $\mathcal{L} \in \mathcal{F}(\mathbb{R})$ representing evaluated OFN. Quite a new fact for evaluation is that for any negatively oriented OFN, its oriented energy index is negative and for any positively oriented OFN, its oriented energy index is positive. Moreover, it is obvious that for any OFN we have

$$
d\left(\overleftrightarrow{\mathcal{L}}\left(a, b, c, d, S_{L}, E_{L}\right)\right)=\left|a\left(\stackrel{\leftrightarrow}{\mathcal{L}}\left(a, b, c, d, S_{L}, E_{L}\right)\right)\right|
$$

This means that oriented energy index stores the information on energy index and the orientation of assessed OFN. This gives new perspectives for imprecision management. 
Example 5. Let us calculate oriented energy index: for OFNs $\overleftrightarrow{\mathcal{U}}$ determined by (18), for $\overleftrightarrow{\mathcal{V}}$ determined by (32), and for $\stackrel{\leftrightarrow}{\mathcal{Z}}=\overleftrightarrow{\mathcal{U}}+\overleftrightarrow{\mathcal{V}}$ determined by (42). Implementing (53), we get the following:

$$
\begin{array}{r}
a(\overleftrightarrow{\mathcal{U}})=\int_{2}^{4} \frac{2 x-4}{x} d x+\int_{4}^{8} d x+\int_{8}^{10} \frac{3 x-30}{x-14} d x \approx 6.3614 \\
a(\overleftrightarrow{\mathcal{V}})=\int_{15}^{11} \frac{3 x-45}{x-23} d x+\int_{11}^{6} d x+\int_{6}^{5} \frac{3 x-15}{x-3} d x \approx 7.8362 \\
a\left(\stackrel{\mathcal{Z}}{x}=\int_{17}^{15} \frac{5(x-19)+\sqrt{x^{2}+10 x-359}}{2(x-23)} d x+\int_{15}^{14} d x \approx-2.0414\right.
\end{array}
$$

An increase in the indistinctness of an OFN suggests that the differences between recommended and unrecommended decision alternatives are harder to differentiate. This leads to an increase in a risk of choosing a not recommended option. Therefore, increase in the indistinctness of OFN implies the decrease in the utility of information described by OFN. The right tool for measuring the indistinctness is the entropy measure, proposed also by de Luca and Termini [35] and modified by Piasecki [36]. In this article, the entropy measure $e \in[0 ; 1]^{\mathcal{F}(\mathbb{R})}$ will be described like in Kosko [45]. For an arbitrary OFN $\stackrel{\leftrightarrow}{\mathcal{L}}\left(a, b, c, d, s_{L}, E_{L}\right) \notin \mathbb{R}$, we have

$$
e\left(\stackrel{\mathcal{L}}{\mathcal{L}}\left(a, b, c, d, S_{L}, E_{L}\right)\right)=\frac{m\left(\mathcal{L} \cap \mathcal{L}^{C}\right)}{m\left(\left(\mathcal{L} \cup \mathcal{L}^{C}\right) \cap[a, d]\right)}
$$

in which the symbol $\mathcal{L}^{C}$ denotes the complement of the fuzzy subset $\mathcal{L} \in \mathcal{F}(\mathbb{R})$ describing evaluated OFN. Due to a good synthetic substantiation and universalism of the above-mentioned formula, the entropy measure proposed by Kosko [45] is now widely used.

Example 6. Let us calculate entropy measure: for OFNs $\overleftrightarrow{\mathcal{U}}$ determined by (18), for $\overleftrightarrow{\mathcal{V}}$ determined by (32), and for $\stackrel{\leftrightarrow}{\mathcal{Z}}=\overleftrightarrow{\mathcal{U}}+\overleftrightarrow{\mathcal{V}}$ determined by (42). Using (58), we get the following:

$$
\begin{gathered}
m\left(\mathcal{U} \cap \mathcal{U}^{C}\right)=\int_{2}^{10} \min \left\{\mu_{\overleftrightarrow{\mathcal{U}}}(x), 1-\mu_{\mathfrak{U}}(x)\right\} d x \\
=\int_{2}^{\frac{8}{3}} \frac{2 x-4}{x} d x+\int_{\frac{8}{3}}^{4}\left(1-\frac{2 x-4}{x}\right) d x+\int_{4}^{8} 0 d x+\int_{8}^{\frac{8}{5}}\left(1-\frac{3 x-30}{x-14}\right) d x+\int_{\frac{46}{5}}^{10} \frac{3 x-30}{x-14} d x \approx 0.9609, \\
m\left(\mathcal{U} \cup \mathcal{U}^{C}\right)=\int_{2}^{10} \max \left\{\mu_{\overleftrightarrow{\mathcal{U}}}(x), 1-\mu_{\overleftrightarrow{\mathcal{U}}}(x)\right\} d x \\
=\int_{2}^{\frac{8}{3}}\left(1-\frac{2 x-4}{x}\right) d x+\int_{\frac{8}{3}}^{4} \frac{2 x-4}{x} d x+\int_{4}^{8} d x+\int_{8}^{\frac{46}{5}} \frac{3 x-30}{x-14} d x+\int_{\frac{46}{5}}^{10}\left(1-\frac{3 x-30}{x-14}\right) d x \approx 7.0391, \\
e(\overleftrightarrow{\mathcal{U}})=\frac{m\left(\mathcal{U} \cap \mathcal{U}^{C}\right)}{m\left(\mathcal{U} \cup \mathcal{U}^{C}\right)} \approx 0.1365 .
\end{gathered}
$$

In analogous way we obtain

$$
e(\overleftrightarrow{\mathcal{V}}) \approx 0.1396, e(\overleftrightarrow{\mathcal{Z}}) \approx 0.2041
$$

The values of oriented energy index entropy measure obtained in Examples 5 and 6 will be used in approximation tasks presented in a following chapter. As it can be easily proved, in case of 
$\operatorname{TrOFN} \stackrel{\leftrightarrow}{\operatorname{Tr}}(a, b, c, d), \notin \mathbb{R}$-oriented energy index and entropy measure are determined basing on the following relation

$$
\begin{aligned}
& a(\overleftrightarrow{\operatorname{Tr}}(a, b, c, d))=\frac{1}{2}(d+c-b-a) \\
& e(\overleftrightarrow{\operatorname{Tr}}(a, b, c, d))=\frac{d-c+b-a}{3 \cdot d+c-b-3 \cdot a}
\end{aligned}
$$

Hence, for TOFN $\stackrel{\leftrightarrow}{\operatorname{Tr}}(a, b, c) \notin \mathbb{R}$, we have

$$
\begin{gathered}
a(\overleftrightarrow{T}(a, b, c))=\frac{1}{2}(d-c), \\
e(\overleftrightarrow{T}(a, b, c))=\frac{1}{3} .
\end{gathered}
$$

From the point of view of real objects modelling, steadiness of indistinctness is a drawback of TOFN. This disadvantage is the result of a lack of any possibility to track the influence of occurring changes on the imprecision element. It is yet another premise to prefer TrOFN as a tool to describe imprecise numbers.

Finally, let's notice the following, simple characteristics of TrOFN.

Lemma 1. For an arbitrary $\operatorname{TrOFN} \stackrel{\leftrightarrow}{\operatorname{Tr}}(a, b, c, d) \notin \mathbb{R}$, we have:

$$
\begin{gathered}
e(\stackrel{\leftrightarrow}{\operatorname{Tr}}(a, b, c, d)) \leq \frac{1}{3} \\
e(\stackrel{\leftrightarrow}{\operatorname{Tr}}(a, b, c, d))=\frac{1}{3} \Leftrightarrow \overleftrightarrow{\operatorname{Tr}}(a, b, c, d)=\stackrel{\leftrightarrow}{T}(a, b, d)=\stackrel{\leftrightarrow}{T}(a, c, d) .
\end{gathered}
$$

Proof. For positively oriented $\operatorname{TrOFN} \overleftrightarrow{\operatorname{Tr}}(a, b, c, d)$, we have $c \geq b$, which implies

$$
e(\overleftrightarrow{\operatorname{Tr}}(a, b, c, d))=\frac{d-c+b-a}{3 \cdot d+c-b-3 \cdot a}=\frac{-d+c-b+a}{-3 \cdot d-c+b+3 \cdot a}=\frac{(a-d)+(c-b)}{3 \cdot(a-d)-(c-b)} \leq \frac{(a-d)}{3 \cdot(a-d)}=\frac{1}{3} .
$$

For negatively oriented $\operatorname{TrOFN} \stackrel{\leftrightarrow}{\operatorname{Tr}}(a, b, c, d)$, we have $b \geq c$, which implies

$$
e(\stackrel{\leftrightarrow}{\operatorname{Tr}}(a, b, c, d))=\frac{d-c+b-a}{3 \cdot d+c-b-3 \cdot a}=\frac{(d-a)+(b-c)}{3 \cdot(d-a)-(b-c)} \leq \frac{(d-a)}{3 \cdot(d-a)}=\frac{1}{3}
$$

The condition (58) follows immediately from the identities (64) and (66).

This conclusion will be used in the next chapter when examining the characteristics of a suggested approximation model of any OFN utilizing TrOFN.

\section{Approximation Problem}

After reference [33], we understand imprecision as a superposition of ambiguity and indistinctness of information. Ambiguity can be interpreted as a lack of a clear recommendation between one alternative among various others. Indistinctness is understood as a lack of explicit distinction between recommended and unrecommended alternatives.

To estimate the distance between any pair of OFNs, we introduce a pseudo-metrics $\delta: \mathbb{K}^{2} \rightarrow \mathbb{R}_{0}^{+}$ determined by a following identity:

$$
\begin{aligned}
& \delta\left(\stackrel{\leftrightarrow}{\mathcal{L}}\left(a_{1}, b_{1}, c_{1}, d_{1}, S_{L}^{(1)}, E_{L}^{(1)}\right), \stackrel{\leftrightarrow}{\mathcal{L}}\left(a_{2}, b_{2}, c_{2}, d_{2}, S_{L}^{(2)}, E_{L}^{(2)}\right)\right) \\
& =\sqrt{\left(a_{1}-a_{2}\right)^{2}+\left(b_{1}-b_{2}\right)^{2}+\left(c_{1}-c_{2}\right)^{2}+\left(d_{1}-d_{2}\right)^{2}}
\end{aligned}
$$


In this section, we will consider the approximation problem of an arbitrary $\mathrm{OFN} \stackrel{\leftrightarrow}{\mathcal{L}}\left(a, b, c, d, S_{L}, E_{L}\right)$ by the nearest $\operatorname{TrOFN} \overleftrightarrow{\operatorname{Tr}}(p, q, r, s)$. Hence, the main criterion of approximation is to determine such $\operatorname{TrOFN} \stackrel{\leftrightarrow}{\operatorname{Tr}}\left(p_{0}, q_{0}, r_{0}, s_{0}\right)$, which will satisfy the following condition:

$$
\begin{gathered}
\delta\left(\overleftrightarrow{\operatorname{Tr}}\left(p_{0}, q_{0}, r_{0}, s_{0}\right), \overleftrightarrow{\mathcal{L}}\left(a, b, c, d, S_{L}, E_{L}\right)\right) \\
=\min \left\{\delta\left(\stackrel{\leftrightarrow}{\operatorname{Tr}}(p, q, r, s), \overleftrightarrow{\mathcal{L}}\left(a, b, c, d, S_{L}, E_{L}\right)\right): \overleftrightarrow{\operatorname{Tr}}(p, q, r, s) \in \mathbb{K}_{T r}\right\} .
\end{gathered}
$$

Function $\delta:\left(\mathbb{K}_{T r}\right)^{2} \rightarrow \mathbb{R}_{0}^{+}$is a metric in $\mathbb{K}_{T r}$. It implies that if any $\stackrel{\leftrightarrow}{\mathcal{L}}\left(a, b, c, d, S_{L}, E_{L}\right) \in \mathbb{K}_{T r}$ then it is the unique solution of the minimization problem (72). Moreover, any crisp number $a \in \mathbb{R}$ is represented by $\operatorname{TrOFN} \overleftrightarrow{\operatorname{Tr}}(a, a, a, a)$. Therefore, we must consider a case of

$$
\overleftrightarrow{\mathcal{L}}\left(a, b, c, d, S_{L}, E_{L}\right) \notin \mathbb{K}_{T r} \supset \mathbb{R}
$$

The condition (72) will be equivalent to a problem

$$
\Phi\left(p_{0}, q_{0}, r_{0}, s_{0} \mid a, b, c, d\right)=\min \left\{\Phi(p, q, r, s \mid a, b, c, d):(p, q, r, s) \in \mathbb{R}^{4}\right\}
$$

of minimization of the objective function $\Phi(\cdot \mid a, b, c, d): \mathbb{R}^{4} \rightarrow \mathbb{R}_{0}^{+}$given by an identity

$$
\Phi(p, q, r, s \mid a, b, c, d)=(p-a)^{2}+(q-b)^{2}+(r-c)^{2}+(s-d)^{2} .
$$

When processing imprecise values, we use FN or OFN only to follow the influence of initial values imprecision on the imprecision of obtained information. Due to our approximation problem, we can impose the requirements of approximating by such value that retains the measures of the imprecision of an approximated value. Using (53) and (58) in an uncomplicated way, we can determine the estimates of ambiguity and indistinctness of the approximated value

$$
\begin{aligned}
& A=a\left(\stackrel{\leftrightarrow}{\mathcal{L}}\left(a, b, c, d, S_{L}, E_{L}\right)\right), \\
& E=e\left(\stackrel{\mathcal{L}}{ }\left(a, b, c, d, S_{L}, E_{L}\right)\right) .
\end{aligned}
$$

In this case, the conditions of imprecision we denote as

$$
\begin{aligned}
& a(\stackrel{\leftrightarrow}{\operatorname{Tr}}(p, q, r, s))=A, \\
& e(\overleftrightarrow{\operatorname{Tr}}(p, q, r, s))=E .
\end{aligned}
$$

Let us also notice that due to the condition (78), OFNs $\stackrel{\leftrightarrow}{\mathcal{L}}\left(a, b, c, d, S_{L}, E_{L}\right)$ i $\overleftrightarrow{\operatorname{Tr}}(p, q, r, s)$ are identically oriented. Juxtaposing the identity (63) and the condition (78) implies a linear equation describing invariance of ambiguity

$$
s+r-q-p=2 \cdot A .
$$

Pairing the identity (50) and the condition (79) implies a linear equation describing invariance of indistinctness

$$
(1-3 \cdot E) \cdot s-(1+E) \cdot r+(1+E) \cdot q-(1-3 \cdot E) \cdot p=0 .
$$

According to Lemma 1, the condition (81) can be used only in case of OFN $\stackrel{\leftrightarrow}{\mathcal{L}}\left(a, b, c, d, S_{L}, E_{L}\right)$ satisfying the condition

$$
e\left(\stackrel{\leftrightarrow}{\mathcal{L}}\left(a, b, c, d, S_{L}, E_{L}\right)\right)=E \leq \frac{1}{3}
$$


For any OFN $\stackrel{\leftrightarrow}{\mathcal{L}}\left(a, b, c, d, S_{L}, E_{L}\right)$ represented, inter alia, by its membership function $\mu_{\mathcal{L}} \in[0,1]^{\mathbb{R}}$, we determine its support closure $\left[\overleftrightarrow{\mathcal{L}}\left(a, b, c, d, S_{L}, E_{L}\right)\right]_{0^{+}}$in the following way

$$
\left[\overleftrightarrow{\mathcal{L}}\left(a, b, c, d, S_{L}, E_{L}\right)\right]_{0^{+}}=\lim _{\alpha \rightarrow 0^{+}}\left\{x \in \mathbb{R}: \mu_{\mathcal{L}}(x) \geq \alpha\right\}=[a, d]
$$

The support closure $\left[\overleftrightarrow{\mathcal{L}}\left(a, b, c, d, S_{L}, E_{L}\right)\right]_{0^{+}}$is interpreted as the smallest closed subset containing all possible values represented by $\operatorname{OFN} \stackrel{\leftrightarrow}{\mathcal{L}}\left(a, b, c, d, S_{L}, E_{L}\right)$. Hence, Invariant Criterion

$$
[a, d]=\left[\overleftrightarrow{\mathcal{L}}\left(a, b, c, d, S_{L}, E_{L}\right)\right]_{0^{+}}=[\overleftrightarrow{\operatorname{Tr}}(p, q, r, s)]_{0^{+}}=[p, s]
$$

can be treated as yet another constraint imposed on approximation method. This Criterion is represented by the equation system:

$$
\left\{\begin{array}{l}
p=a \\
s=d
\end{array}\right.
$$

Each TrOFN feasible in the approximation task will be called a feasible TrOFN. Conditions (80), (81), and (85) are restrictions that limit a set of feasible TrOFN. The solution of OFN approximation is the nearest feasible TrOFN, denoted by the symbol $\stackrel{\leftrightarrow}{T_{0}}$. In following subsections, we will analyse chosen problems of approximation OFN by TrOFN. Each of those problems will be distinguished by a chosen combination of restrictions limiting the set of feasible TrOFN. Each of approximation problems is called $X Y Z$-approximation, in which the prefix $X Y Z$ is an acronym identifying the approximation problem. The solution of XYZ-approximation of any OFN $\overleftrightarrow{\mathcal{L}}\left(a, b, c, d, S_{L}, E_{L}\right)$ is denoted by a symbol $\overleftrightarrow{\operatorname{Tr}}_{X Y Z}\left(\stackrel{\leftrightarrow}{\mathcal{L}}\left(a, b, c, d, S_{L}, E_{L}\right)\right)$

\subsection{Unconditional Approximation (UC-Approximation)}

Initially, we will consider the approximation problem with no constraints imposed on the set of feasible TrOFN. This approximation problem is called UC-approximation. UC-approximation is determined just by the objective function (74). In the face of a lack of any restricting equations, the solution of UC-approximation problem of OFN $\stackrel{\leftrightarrow}{\mathcal{L}}\left(a, b, c, d, S_{L}, E_{L}\right)$ is TrOFN

$$
\stackrel{\leftrightarrow}{\operatorname{Tr}}(a, b, c, d)=\overleftrightarrow{\operatorname{Tr}}_{U C}\left(\stackrel{\leftrightarrow}{\mathcal{L}}\left(a, b, c, d, S_{L}, E_{L}\right)\right)
$$

It is obvious that a UC-approximation problem always has a solution.

Example 7. For OFNs $\overleftrightarrow{\mathcal{U}}=\overleftrightarrow{\mathcal{L}}\left(2,4,8,10, S_{U}, E_{U}\right)$ determined by (18), $\overleftrightarrow{\mathcal{V}}=\overleftrightarrow{\mathcal{L}}\left(15,11,6,5, S_{V}, E_{V}\right)$

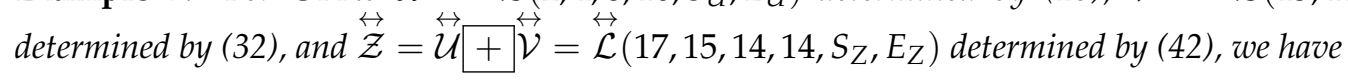

$$
\stackrel{\leftrightarrow}{\operatorname{Tr}}(2,4,8,10)=\overleftrightarrow{\operatorname{Tr}}_{U C}(\overleftrightarrow{\mathcal{U}}), \stackrel{\leftrightarrow}{\operatorname{Tr}}(15,11,6,5)=\overleftrightarrow{\operatorname{Tr}}_{U C}(\overleftrightarrow{\mathcal{V}}), \stackrel{\leftrightarrow}{\operatorname{Tr}}(17,15,14,14)=\overleftrightarrow{\operatorname{Tr}}_{U C}(\overleftrightarrow{\mathcal{Z}})
$$

Then, we evaluate an example influence that UC-approximation had on the imprecision measure. For OFNs $\overleftrightarrow{\mathcal{U}}, \overleftrightarrow{\mathcal{V}}$, and $\overleftrightarrow{\mathcal{Z}}$, the results gathered in Examples 5 and 6 were used. In case of $\operatorname{TrOFNs} \overleftrightarrow{\operatorname{Tr}}_{U C}(\overleftrightarrow{\mathcal{U}}), \overleftrightarrow{\operatorname{Tr}}_{U C}(\overleftrightarrow{\mathcal{V}})$, and $\overleftrightarrow{T r}_{U C}(\overleftrightarrow{\mathcal{Z}})$, the imprecision characteristics were determined by formulas (63) and (64). All those values are presented in Table 1 . 
Table 1. UC-approximation impact on imprecision evaluation.

\begin{tabular}{|c|c|c|c|c|}
\hline$\stackrel{\leftrightarrow}{\mathcal{L}}$ & $a(\overleftrightarrow{\mathcal{L}})$ & $a\left(\overleftrightarrow{\mathbf{T}}_{U C}(\overleftrightarrow{\mathcal{L}})\right)$ & $e(\overleftrightarrow{\mathcal{L}})$ & $e\left(\stackrel{\operatorname{Tr}}{U C}_{U C}(\stackrel{\leftrightarrow}{\mathcal{L}})\right)$ \\
\hline$\overleftrightarrow{\mathcal{U}}$ & 6.3614 & 6.0000 & 0.1365 & 0.1429 \\
\hline$\overleftrightarrow{\mathcal{V}}$ & -7.8362 & -7.500 & 0.1396 & 0.1429 \\
\hline$\overleftrightarrow{\mathcal{Z}}$ & -2.0414 & -2.000 & 0.2041 & 0.2000 \\
\hline
\end{tabular}

The results presented in this example show that UC-approximation to a certain extent distorts the imprecision evaluations. However, these evaluations are only the values representing the points on conventional scale. Therefore, the observed deviations of less than $5 \%$ of relative error can be considered insignificant.

On the other hand, the comparison of identities (15) and (45) lets us conclude that for any pair $(\beta, \overleftrightarrow{\mathcal{M}}) \in \mathbb{R} \times \mathbb{K}$ we have

$$
\beta \odot \overleftrightarrow{\operatorname{Tr}}_{U C}(\overleftrightarrow{\mathcal{M}})=\overleftrightarrow{\operatorname{Tr}}_{U C}(\beta \odot \overleftrightarrow{\mathcal{M}})
$$

Moreover, from the comparison of identities (21)-(27) and (47), it follows that for any pair $(\overleftrightarrow{\mathcal{K}}, \overleftrightarrow{\mathcal{M}}) \in \mathbb{K}^{2}$ we have

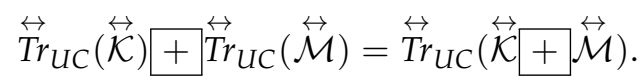

It means that the function $\stackrel{\leftrightarrow}{T}_{U C} \in\left(\mathbb{K}_{T r}\right)^{\mathbb{K}}$ is a linear operator on $\mathbb{K}$. Due to this property, the errors of the estimations of oriented energy index and entropy measure did not succumb to propagation. This is a vital advantage of UC-approximation method.

\subsection{Approximation under Criterion of Constant Ambiguity (CA-Approximation)}

Let us consider the approximation problem in which each feasible TrOFN satisfies the condition (78). Such an approximation task we call CA-approximation problem. Then, the coordinates of all the feasible $\operatorname{TrOFN} \stackrel{\leftrightarrow}{\operatorname{Tr}}(p, q, r, s)$ can be presented as a general solution of the Equation (80). This solution is given as follows

$$
\left\{\begin{array}{c}
p=x, \\
q=y, \\
r=z, \\
s=2 \cdot A+x+y-z,
\end{array} \quad x, y, z \in \mathbb{R},\right.
$$

in which the sequence $(x, y, z)$ is a monotonic sequence. Then-to solve the OFN $\overleftrightarrow{\mathcal{L}}\left(a, b, c, d, S_{L}, E_{L}\right)$ CA-approximation problem - the first step is to find the minimum of a function

$$
\varphi(x, y, z)=\Phi(x, y, z, 2 \cdot A+x+y-z \mid a, b, c, d) .
$$

The minimum is reached in a point $\left(x_{0}, y_{0}, z_{0}\right)$ of following coordinates

$$
\left\{\begin{array}{l}
x_{0}=\frac{3 \cdot a-b+c+d-2 \cdot A}{4} \\
y_{0}=\frac{-a+3 \cdot b+c+d-2 \cdot A}{4} \\
z_{0}=\frac{a+b+3 \cdot c-d+2 \cdot A}{4} .
\end{array}\right.
$$

When inserting those coordinates to (90), we conclude that the solution of OFN $\stackrel{\leftrightarrow}{\mathcal{L}}\left(a, b, c, d, S_{L}, E_{L}\right)$ CA-approximation problem is TrOFN

$$
\overleftrightarrow{\operatorname{Tr}}\left(\frac{3 \cdot a-b+c+d-2 \cdot A}{4}, \frac{-a+3 \cdot b+c+d-2 \cdot A}{4}, \frac{a+b+3 \cdot c-d+2 \cdot A}{4}, \frac{a+b-c+3 \cdot d+2 \cdot A}{4}\right)=\overleftrightarrow{\operatorname{Tr}}_{C A}\left(\overleftrightarrow{\mathcal{L}}\left(a, b, c, d, S_{L}, E_{L}\right)\right)
$$


Example 8. For OFNs $\overleftrightarrow{\mathcal{U}}=\overleftrightarrow{\mathcal{L}}\left(2,4,8,10, S_{U}, E_{U}\right)$ determined by (18), $\overleftrightarrow{\mathcal{V}}=\overleftrightarrow{\mathcal{L}}\left(15,11,6,5, S_{V}, E_{V}\right)$ determined by (32), and $\overleftrightarrow{\mathcal{Z}}=\overleftrightarrow{\mathcal{U}}+\overleftrightarrow{\mathcal{V}}=\overleftrightarrow{\mathcal{L}}\left(17,15,14,14, S_{Z}, E_{Z}\right)$ determined by (42), we have

$$
\begin{gathered}
\overleftrightarrow{\operatorname{Tr}}(1.8193,3.8193,8.1807,10.1807)=\overleftrightarrow{\operatorname{Tr}}_{C A}(\overleftrightarrow{\mathcal{U}}) \\
\overleftrightarrow{\operatorname{Tr}}(15.1681,11.1681,5.8319,4.8319)=\overleftrightarrow{\operatorname{Tr}}_{C A}(\overleftrightarrow{\mathcal{V}}) \\
\overleftrightarrow{\operatorname{Tr}}(17.0207,15.0207,13.9793,13.9793)=\overleftrightarrow{\operatorname{Tr}}_{C A}(\overleftrightarrow{\mathcal{Z}})
\end{gathered}
$$

Next, we assess an example influence that CA-approximation had on indistinctness evaluation. For OFNs $\overleftrightarrow{\mathcal{U}}, \overleftrightarrow{\mathcal{V}}, \overleftrightarrow{\mathcal{Z}}, \overleftrightarrow{\operatorname{Tr}}_{U C}(\overleftrightarrow{\mathcal{U}}), \overleftrightarrow{\operatorname{Tr}}_{U C}(\overleftrightarrow{\mathcal{V}})$, and $\overleftrightarrow{T r}_{U C}(\overleftrightarrow{\mathcal{Z}})$, the results from Example 7 were used. In case of TrOFNs $\overleftrightarrow{\operatorname{Tr}}_{C A}(\overleftrightarrow{\mathcal{U}}), \overleftrightarrow{T r}_{C A}(\overleftrightarrow{\mathcal{V}})$, and $\overleftrightarrow{\operatorname{Tr}}_{C A}(\overleftrightarrow{\mathcal{Z}})$, their entropy measure was determined by the formula (64). All these values

\begin{tabular}{|c|c|c|c|}
\hline$\stackrel{\leftrightarrow}{\mathcal{L}}$ & $e(\stackrel{\leftrightarrow}{\mathcal{L}})$ & $\boldsymbol{e}\left(\stackrel{\leftrightarrow}{\boldsymbol{T}}_{C A}(\stackrel{\leftrightarrow}{\mathcal{L}})\right)$ & $e\left(\stackrel{\operatorname{Tr}}{U C}_{U C}(\overleftrightarrow{\mathcal{L}})\right)$ \\
\hline$\overleftrightarrow{\mathcal{U}}$ & 0.1365 & 0.1358 & 0.1429 \\
\hline$\overleftrightarrow{\mathcal{V}}$ & 0.1396 & 0.1375 & 0.1429 \\
\hline$\overleftrightarrow{\mathcal{Z}}$ & 0.2041 & 0.1967 & 0.2000 \\
\hline
\end{tabular}
are presented in Table 2.

Table 2. CA-approximation impact on indistinctness evaluation.

Source: Own elaboration.

Moreover, we have here

$$
\begin{aligned}
& {\stackrel{\leftrightarrow}{\operatorname{Tr}_{C A}}}_{C}(\overleftrightarrow{\mathcal{U}})+\stackrel{\leftrightarrow}{\operatorname{Tr}}_{C A}(\overleftrightarrow{\mathcal{V}})=\overleftrightarrow{\operatorname{Tr}}(1.8193,3.8193,8.1807,10.1807)+\stackrel{\leftrightarrow}{\operatorname{Tr}}(15.1681,11.1681,5.8319,4.8319) \\
& =\stackrel{\leftrightarrow}{\operatorname{Tr}}(16.9874,14.9874,14.0126,14.0126) \neq \stackrel{\leftrightarrow}{\operatorname{Tr}}(17.0207,15.0207,13.9793,13.9793)=\overleftrightarrow{\operatorname{Tr}}_{C A}(\stackrel{\leftrightarrow}{\mathcal{Z}}) .
\end{aligned}
$$

Hence, the function $\stackrel{\leftrightarrow}{T}_{A C} \in\left(\mathbb{K}_{T r}\right)^{\mathbb{K}}$ is not an additive operator on $\mathbb{K}$. Furthermore, the two equations below show the existing effect of estimation errors propagation of oriented energy index and entropy measure.

$$
\begin{gathered}
a\left(\overleftrightarrow{\operatorname{Tr}}_{C A}(\overleftrightarrow{\mathcal{U}})+\overleftrightarrow{\operatorname{Tr}}_{C A}(\overleftrightarrow{\mathcal{V}})\right)=-1.9784 \neq-2.0414=a\left(\overleftrightarrow{\operatorname{Tr}}_{C A}(\overleftrightarrow{\mathcal{Z}})\right) \\
e\left(\overleftrightarrow{\operatorname{Tr}}_{C A}(\overleftrightarrow{\mathcal{U}})+\overleftrightarrow{\operatorname{Tr}}_{C A}(\overleftrightarrow{\mathcal{V}})\right)=0.2020 \neq 0.1967=e\left(\overleftrightarrow{\operatorname{Tr}}_{C A}(\overleftrightarrow{\mathcal{Z}})\right) .
\end{gathered}
$$

The above example shows that the influence of CA-approximation on the entropy measure evaluation is moderate, and when it comes to its scale it is similar to the influence of UC-approximation method. This example proves that the function $\stackrel{\leftrightarrow}{T r}_{C A} \in\left(\mathbb{K}_{T r}\right)^{\mathbb{K}}$ is not a linear operator on $\mathbb{K}$. Calculations run in this example indicate the possibility of the occurrence of the error estimation propagation of oriented energy index and entropy measure. An extremely interesting phenomenon is revealed by Equation (98). IT appears that during the addition of OFNs approximations with precisely imitated ambiguity index, the error propagation of index estimation occurs. This phenomenon will be further called a zero-error propagation phenomenon.

\subsection{Approximation under Criterion of Constant Imprecision (CI-Approximation)}

Let us consider an approximation problem in which each feasible TrOFN satisfies conditions (78) and (79). Such a task will be called CI-approximation. Then, coordinates of all feasible TrOFN 
$\stackrel{\leftrightarrow}{\operatorname{Tr}}(p, q, r, s)$ can be presented as a general solution of equation system (80) and (81). This solution is given as

$$
\left\{\begin{array}{c}
p=x \\
q=y, \\
r=y+\frac{1-3 \cdot E}{1-E} \cdot A, \\
s=x+\frac{1+E}{1-E} \cdot A
\end{array} \quad x, y \in \mathbb{R} .\right.
$$

Then, to solve the OFN $\stackrel{\leftrightarrow}{\mathcal{L}}\left(a, b, c, d, S_{L}, E_{L}\right)$ CI-approximation problem, the first step is to find the minimum of a function

$$
\varphi(x, y)=\Phi\left(x, y, y+\frac{1-3 \cdot E}{1-E} \cdot A, x+\frac{1+E}{1-E} \cdot A \mid a, b, c, d\right)
$$

This minimum is reached in a point $\left(x_{0}, y_{0}\right)$ of following coordinates

$$
\left\{\begin{array}{l}
x_{0}=\frac{a+d}{2}-\frac{1+E}{2 \cdot(1-E)} \cdot A, \\
y_{0}=\frac{b+c}{2}-\frac{1-3 \cdot E}{2 \cdot(1-E)} \cdot A .
\end{array}\right.
$$

After inserting these coordinates to (98), we can conclude that the solution of CI-approximation problem of OFN $\overleftrightarrow{\mathcal{L}}\left(a, b, c, d, S_{L}, E_{L}\right)$ fulfilling condition (82) is TrOFN

$$
\overleftrightarrow{\operatorname{Tr}}\left(\frac{a+d}{2}-\frac{1+E}{2 \cdot(1-E)} \cdot A, \frac{b+c}{2}-\frac{1-3 \cdot E}{2 \cdot(1-E)} \cdot A, \frac{b+c}{2}+\frac{1-3 \cdot E}{2 \cdot(1-E)} \cdot A, \frac{a+d}{2}+\frac{1+E}{2 \cdot(1-E)} \cdot A\right)=\overleftrightarrow{\operatorname{Tr}}_{C I}\left(\overleftrightarrow{\mathcal{L}}\left(a, b, c, d, S_{L}, E_{L}\right)\right) .
$$

Example 9. For OFNs $\overleftrightarrow{\mathcal{U}}=\overleftrightarrow{\mathcal{L}}\left(2,4,8,10, S_{U}, E_{U}\right)$ determined by (18), $\overleftrightarrow{\mathcal{V}}=\overleftrightarrow{\mathcal{L}}\left(15,11,6,5, S_{V}, E_{V}\right)$ determined by (32), and $\overleftrightarrow{\mathcal{Z}}=\overleftrightarrow{\mathcal{U}}+\overleftrightarrow{\mathcal{V}}=\overleftrightarrow{\mathcal{L}}\left(17,15,14,14, S_{Z}, E_{Z}\right)$ determined by (42), we have

$$
\begin{gathered}
\overleftrightarrow{\operatorname{Tr}}(1.8137,3.8248,8.1751,10.1863)=\overleftrightarrow{\operatorname{Tr}}_{C I}(\overleftrightarrow{\mathcal{U}}), \\
\overleftrightarrow{\operatorname{Tr}}(15.1610,11.1752,5.8248,4.8390)=\overleftrightarrow{\operatorname{Tr}}_{C I}(\overleftrightarrow{\mathcal{V}}) \\
\overleftrightarrow{\operatorname{Tr}}(17.0442,14.9972,14.0038,13.9558)=\overleftrightarrow{\operatorname{Tr}}_{C I}(\overleftrightarrow{\mathcal{Z}})
\end{gathered}
$$

Furthermore, we have here

$$
\begin{aligned}
& \stackrel{\leftrightarrow}{\operatorname{Tr}}_{C I}(\overleftrightarrow{\mathcal{U}})+\stackrel{\leftrightarrow}{\operatorname{Tr}}_{C I}(\stackrel{\leftrightarrow}{\mathcal{V}})=\overleftrightarrow{\operatorname{Tr}}(1.8137,3.8248,8.1751,10.1863)+\stackrel{\leftrightarrow}{\operatorname{Tr}}(15.1610,11.1752,5.8248,4.8390) \\
& =\overleftrightarrow{\operatorname{Tr}}(16.9747,15.0000,13.9999,13.9999) \neq \overleftrightarrow{\operatorname{Tr}}(17.0442,14.9972,14.0038,13.9558)=\overleftrightarrow{\operatorname{Tr}}_{C I}(\overleftrightarrow{\mathcal{Z}}) .
\end{aligned}
$$

Therefore, the function $\stackrel{\leftrightarrow}{\operatorname{Tr}}_{C I} \in\left(\mathbb{K}_{T r}\right)^{\mathbb{K}}$ is not an additive operator on $\mathbb{K}$. Moreover, the two equations presented below are examples of an occurrence of zero-error propagation phenomenon.

$$
\begin{gathered}
a\left(\stackrel{\operatorname{Tr}}{C I}_{C I}(\overleftrightarrow{\mathcal{U}})+\stackrel{\leftrightarrow}{T}_{C I}(\overleftrightarrow{\mathcal{V}})\right)=-1.9875 \neq-2.0414=a\left(\overleftrightarrow{\operatorname{Tr}}_{C I}(\overleftrightarrow{\mathcal{Z}})\right) \\
e\left(\overleftrightarrow{\operatorname{Tr}}_{C I}(\overleftrightarrow{\mathcal{U}})++\overleftrightarrow{\operatorname{Tr}}_{C I}(\overleftrightarrow{\mathcal{V}})\right)=0.1990 \neq 0.2041=e\left(\overleftrightarrow{T r}_{C I}(\overleftrightarrow{\mathcal{Z}})\right) .
\end{gathered}
$$

The above example proves that the function $\stackrel{\leftrightarrow}{\operatorname{Tr}}_{C I} \in\left(\mathbb{K}_{T r}\right)^{\mathbb{K}}$ is not a linear operator on $\mathbb{K}$. The calculations in this example confirm that there is a possibility of an occurrence of zero-error propagation phenomenon of oriented energy index and entropy measure estimations. 


\subsection{Approximation under Invariant Criterion (IC-Approximation)}

Let us consider an approximation problem in which each feasible TrOFN satisfies conditions (84). Such an approximation task will be called IC-approximation. Then, coordinates of all feasible TrOFN $\overleftrightarrow{\operatorname{Tr}}(p, q, r, s)$ can be presented as a general solution of the Equation (85). This solution is given as

$$
\left\{\begin{array}{l}
p=a \\
q=x, \\
r=y, \\
s=d
\end{array}\right.
$$

Then, to solve the OFN $\stackrel{\leftrightarrow}{\mathcal{L}}\left(a, b, c, d, S_{L}, E_{L}\right)$ IC-approximation problem, the first step is to find the minimum of a function

$$
\varphi(x, y)=\Phi(a, x, y, d \mid a, b, c, d) .
$$

This minimum is reached in a point $\left(x_{0}, y_{0}\right)$ of following coordinates

$$
\left\{\begin{array}{l}
x_{0}=b \\
y_{0}=c
\end{array}\right.
$$

In this instance, we can conclude that the solution of IC-approximation problem of OFN $\overleftrightarrow{\mathcal{L}}\left(a, b, c, d, S_{L}, E_{L}\right)$ is TrOFN

$$
\overleftrightarrow{\operatorname{Tr}}(a, b, c, d)=\overleftrightarrow{\operatorname{Tr}}_{I C}\left(\stackrel{\leftrightarrow}{\mathcal{L}}\left(a, b, c, d, S_{L}, E_{L}\right)\right)=\overleftrightarrow{\operatorname{Tr}}_{U C}\left(\overleftrightarrow{\mathcal{L}}\left(a, b, c, d, S_{L}, E_{L}\right)\right)
$$

This means that UC-approximation and IC-approximation problems always have an identical solution. Therefore, there is no need to consider IC-approximation problem.

\subsection{Approximation under Invariant Criterion with Constant Ambiguity (ICCA-Approximation)}

Let us consider an approximation problem in which each feasible TrOFN satisfies conditions (78) and (84). Such an approximation task will be called ICCA-approximation problem. Then, coordinates

of all feasible $\operatorname{TrOFN} \overleftrightarrow{\operatorname{Tr}}(p, q, r, s)$ can be presented as a general solution of equation system (80) and (85). This solution is given as

$$
\left\{\begin{array}{c}
p=a \\
q=x \\
r=x+2 \cdot A+a-d, \quad x \in \mathbb{R} . \\
s=d
\end{array}\right.
$$

Then, to solve the OFN $\stackrel{\leftrightarrow}{\mathcal{L}}\left(a, b, c, d, S_{L}, E_{L}\right)$ ICCA-approximation problem, the first step is to find the minimum of a function

$$
\varphi(x)=\Phi(a, x, x+2 \cdot A+a-d, d \mid a, b, c, d) .
$$

This minimum is reached in a point

$$
x_{0}=\frac{-a+b+c+d}{2}-A .
$$


After inserting that value to (114), we can conclude that the solution of ICCA-approximation problem of OFN $\overleftrightarrow{\mathcal{L}}\left(a, b, c, d, S_{L}, E_{L}\right)$ is TrOFN

$$
\overleftrightarrow{\operatorname{Tr}}\left(a, \frac{-a+b+c+d}{2}-A, \frac{a+b+c-d}{2}+A, d\right)=\overleftrightarrow{\operatorname{Tr}}_{I C C A}\left(\stackrel{\leftrightarrow}{\mathcal{L}}\left(a, b, c, d, S_{L}, E_{L}\right)\right)
$$

Example 10. For OFNs $\overleftrightarrow{\mathcal{U}}=\overleftrightarrow{\mathcal{L}}\left(2,4,8,10, S_{U}, E_{U}\right)$ determined by $(18), \overleftrightarrow{\mathcal{V}}=\overleftrightarrow{\mathcal{L}}\left(15,11,6,5, S_{V}, E_{V}\right)$ determined by (32), and $\overleftrightarrow{\mathcal{Z}}=\overleftrightarrow{\mathcal{U}}+\overleftrightarrow{\mathcal{V}}=\overleftrightarrow{\mathcal{L}}\left(17,15,14,14, S_{Z}, E_{Z}\right)$ determined by (42), we have

$$
\begin{aligned}
\overleftrightarrow{\operatorname{Tr}}(2,3.6386,8.3614,10) & =\overleftrightarrow{\operatorname{Tr}}_{I C C A}(\overleftrightarrow{\mathcal{U}}), \\
\overleftrightarrow{\operatorname{Tr}}(15,11.3362,5.6638,5) & =\overleftrightarrow{\operatorname{Tr}}_{I C C A}(\overleftrightarrow{\mathcal{V}}), \\
\overleftrightarrow{\operatorname{Tr}}(17,15.0414,13.9586,14) & =\overleftrightarrow{\operatorname{Tr}}_{I C C A}(\overleftrightarrow{\mathcal{Z}}) .(!)
\end{aligned}
$$

Let us notice that the sequence $(17,15.0414,13.9586,14)$ is not monotonic. Therefore, the object $\overleftrightarrow{\operatorname{Tr}}(17,15.0414,13.9586,14)$ is not an OFN. It is described by its membership relation $\mu_{\overleftrightarrow{T r}}(\cdot \mid a, b, c, d) \in$ $\mathbb{R} \times[0,1]$ given by the identity (43). This object is an example of an improper OFN described in [6]. Summing up, the solution of an ICCA-approximation problem OFN $\overleftrightarrow{\mathcal{Z}}$ does not exist. In addition, this fact also results in conclusion that for the triple $(\overleftrightarrow{\mathcal{U}}, \overleftrightarrow{\mathcal{V}}, \overleftrightarrow{\mathcal{Z}})$, the function $\overleftrightarrow{T r}_{I C C I} \in\left(\mathbb{K}_{T r}\right)^{\mathbb{K}}$ does not fulfil the condition (89).

Next, we evaluate an example influence that ICCA-approximation had on indistinctness evaluation. For OFNs $\overleftrightarrow{\mathcal{U}}, \overleftrightarrow{\mathcal{V}}, \overleftrightarrow{\mathcal{Z}}, \overleftrightarrow{\operatorname{Tr}}_{C A}(\overleftrightarrow{\mathcal{U}}), \overleftrightarrow{\operatorname{Tr}}_{C A}(\overleftrightarrow{\mathcal{V}}), \overleftrightarrow{\operatorname{Tr}}_{C A}(\overleftrightarrow{\mathcal{Z}}), \overleftrightarrow{\operatorname{Tr}}_{U C}(\overleftrightarrow{\mathcal{U}}), \overleftrightarrow{\operatorname{Tr}}_{U C}(\overleftrightarrow{\mathcal{V}})$, and $\overleftrightarrow{\operatorname{Tr}}_{U C}(\overleftrightarrow{\mathcal{Z}})$, the results from

\begin{tabular}{|c|c|c|c|c|}
\hline$\stackrel{\leftrightarrow}{\mathcal{L}}$ & $e(\stackrel{\mathcal{L}}{)})$ & $e\left(\stackrel{\operatorname{Tr}}{I C C A}_{\text {ICA }}(\stackrel{\leftrightarrow}{\mathcal{L}})\right)$ & $e\left(\overleftrightarrow{\operatorname{Tr}}_{C A}(\stackrel{\leftrightarrow}{\mathcal{L}})\right)$ & $e\left(\overleftrightarrow{\operatorname{Tr}}_{U C}(\overleftrightarrow{\mathcal{L}})\right)$ \\
\hline$\overleftrightarrow{\mathcal{U}}$ & 0.1365 & 0.1141 & 0.1358 & 0.1429 \\
\hline$\overleftrightarrow{\mathcal{V}}$ & 0.1396 & 0.1213 & 0.1375 & 0.1429 \\
\hline$\stackrel{\leftrightarrow}{\mathcal{Z}}$ & 0.2041 & - & 0.1967 & 0.2000 \\
\hline
\end{tabular}
Example 8 were used. In case of TrOFNs $\overleftrightarrow{\operatorname{Tr}}_{\text {ICCA }}(\overleftrightarrow{\mathcal{U}})$ and $\overleftrightarrow{\operatorname{Tr}}_{\text {ICCA }}(\overleftrightarrow{\mathcal{V}})$, their entropy measures were determined based on formula (64). All these values are given in Table 3.

Table 3. ICCA-approximation impact on indistinctness evaluation.

Source: Own elaboration.

The above example proves that the function $\stackrel{\leftrightarrow}{T}_{I C C A} \in\left(\mathbb{K}_{T r}\right)^{\mathbb{K}}$ is not a linear operator on $\mathbb{K}$. The lack of linearity of that function lets us also expect that the zero-error propagation phenomenon will occur. The calculations in this example also prove the thesis that for chosen OFNs, the solution of ICCA-approximation problem might not exist. Similar conclusion can be drawn in case of CA-approximation and CI-approximation.

\subsection{Approximation under Invariant Criterion with Constant Imprecision (ICCI-Approximation)}

Let us consider an approximation problem in which each feasible TrOFN satisfies conditions (78), (79), and (84). Such an approximation task will be called ICCI-approximation problem. Using the substitutions (85), the equation system (80), (81), and (85) can be transformed into the following equivalent equation system:

$$
\left\{\begin{array}{c}
r-q=2 \cdot A-d+a \\
(1+E) \cdot r-(1+E) \cdot q=(1-3 \cdot E) \cdot(-d+a) .
\end{array}\right.
$$


According to Cramer's Rule [46], system of Equation (121) is consistent if it satisfies the following condition:

$$
(2 \cdot A-d+a) \cdot(1+E)=(1-3 \cdot E) \cdot(-d+a),
$$

From the condition (122), we have

$$
E=\frac{d-a}{2 \cdot A-(d-a)}
$$

because the condition (73) implies $a \neq d$. Therefore, there is no need to consider ICCI-approximation problem. On the other hand, we can say that if OFN $\stackrel{\leftrightarrow}{\mathcal{L}}\left(a, b, c, d, S_{L}, E_{L}\right)$ satisfies the conditions (82) and (123) then the ICCA-approximation problem solution $\stackrel{\leftrightarrow}{\operatorname{Tr}}_{I C C A}\left(\stackrel{\leftrightarrow}{\mathcal{L}}\left(a, b, c, d, S_{L}, E_{L}\right)\right)$ additionally satisfies the condition (84).

\section{Recommendations}

OFNs are a form of a record of imprecise information. The purpose of approximation of OFNs by TrOFNs was justified in the paper. Following that premise, six OFN approximation problems by the nearest TrOFN were proposed and discussed in the paper. It appeared that the two remaining approximation problems UC-approximation ad IC-approximation always deliver identical solutions. Additionally, the ICCI-approximation and ICCA-approximation problem always have identical solutions. Therefore, they should not be differentiated. Each of remaining four approximation methods has its advantages and disadvantages.

The main advantage of UC-approximation method is the fact that it is the only discussed method that delivers a solution of any OFN. IT is also the only one of the discussed approximation methods free of the occurrence of error propagation phenomenon of imprecision characteristics, that is, ambiguity index and entropy measure. Thanks to using the UC-approximation for processing complex sets of information, it is always safe. The drawbacks of UC-approximation method are the discrepancies between the characteristics of imprecision of approximated OFN and approximating TrOFN. This distorts the usefulness evaluation of processed information. From the point of view of algebra, UC-approximation is a linear operator of all OFN space.

All other approximation methods might lack the solution of incidental OFN. Such cases can be easily recognised utilising the fact that every TrOFN is characterised by a monotonic sequence of parameters. If, during implementation of a chosen approximation method of a given OFN we will obtain a non-monotonic sequence of parameters, then such an approximation problem has no solution. Attempts should be made to approximate a given OFN using another approximation method. There always exists at least one approximation method with a solution for a given OFN.

CA-approximation method approximates a given OFN by such TrOFN that its oriented energy index is equal to oriented energy index of OFN. This lets us monitor the usefulness of processed information more credibly. A more precise approximation method is ICCS-approximation, which approximates a given OFN by TrOFN of the identical support closure and equally oriented energy index. It allows one to monitor the usefulness of processed information more credibly. This means that CA-approximation should be used only in cases in which ICCA-approximation method does not deliver the solution. For both these approximation methods, there are no universal conditions restraining the set of approximated OFNs.

On the contrary, in case of CI-approximation method, the approximated OFNs must be characterised by a low level of entropy that satisfies condition (82). The CA-approximation method approximated a given OFN by TrOFN with an equally oriented energy index and entropy measure. It lets us monitor the usefulness of processed information more credibly than in case of the approximation obtained by the CA-approximation method.

A significant, common disadvantage of CA-, CI-, and ICCA-approximation methods is the occurrence of zero-error propagation phenomenon, described in Section 4 . This usually leads to the 
conclusion that using such methods of approximation in cases of big, complex sets of information creates a threat for the credible monitoring of processed data usefulness.

In this case, the procedure of approximation method choice depends on individual preferences of the researcher who wants to approximate OFNs by TrOFNs. Nevertheless, it can be clarified by the following procedure:

- If the researcher accepts the phenomenon of zero-error propagation, then one should choose CIor ICCA-approximation method if applicable. Otherwise, one should choose UC-approximation.

- If, in a considered case, the approximation method provides no solution, then the researcher chooses the second of CI- or ICCA-approximation method,

- If, in the considered case, CI- and ICCA-approximation methods do not have solutions, then the researcher should choose the CA-approximation method,

- If, in the considered case, the CA-approximation method does not have a solution, then the researcher should choose UC-approximation.

The above-described procedure of the approximation method choice can be used separately for any number. It is acceptable to use different methods for different numbers in the same empirical implementation.

The results obtained in the paper let us, for instance, use the Oriented Behavioural Present Value $[17,18]$ in portfolio models described in $[19,21,22,24]$.

\section{Conclusions}

The results obtained in this article will facilitate the use of OFN in real object modelling.

In the face of conditions (7) and (8), the above-described approximation methods can be used for approximate FN by trapezoidal FN. The oriented energy index is then reduced to the energy measure.

Among other things, FN are applied in social choice and political sciences. In reference [47], FN describe imprecise linguistic evaluation labels used by voters. Each assessment can be irrationally undervalued or overvalued. Then, we can use OFN to describe the irrational deviations of the assessment. In [48], FN describe voters' opinion profiles. This is a static description of the population of voters. On the other side, voters' opinions are very dynamic. Replacement of FN by OFN would allow one to describe mentioned dynamics. The problems discussed in this paper can be a starting point for an attempt to create such approximation methods of OFN by TrOFN that such a constraint that a "vector of solution parameters is a monotonic sequence" will be included. Moreover, further research on the considered above approximation method should be devoted to the following problems:

- the impact of approximation on the ordering of OFNs,

- the impact of the approximation on a solution to equations determined by OFNs,

- the impact of approximation on formal modelling of real objects.

Solutions obtained to the last three problems will allow estimation of the approximation error.

Author Contributions: Conceptualization, K.P.; Methodology, K.P. and A.Ł.H.; Validation, A.Ł.H.; Formal Analysis, A.Ł.H.; Writing-Original Draft Preparation, K.P.; Writing-Review \& Editing, K.P. and A.Ł.H.

Funding: This research received no external funding.

Acknowledgments: The authors are very grateful to the anonymous reviewers for their insightful and constructive comments and suggestions. We would like to thank to Professor José Carlos R. Alcantud for his valuable final remarks. Using these comments allowed us to improve this article.

Conflicts of Interest: The authors declare no conflict of interest.

\section{References}

1. Kosiński, W.; Słysz, P. Fuzzy numbers and their quotient space with algebraic operations. Bull. Pol. Acad. Sci. 1993, 41, 285-295. 
2. Kosiński, W.; Prokopowicz, P.; Ślęzak, D. Fuzzy reals with algebraic operations: Algorithmic approach. In Advances in Soft Computing, Proceedings of the Intelligent Information Systems 2002 Symposium, Sopot, Poland, 3-6 June 2002; Klopotek, M., Wierzchoń, S.T., Michalewicz, M., Eds.; Springer: Heidelberg, Germany, 2002; pp. 311-320.

3. Kosiński, W.; Prokopowicz, P.; Ślęzak, D. Ordered fuzzy numbers. Bull. Pol. Acad. Sci. 2003, 51, 327-339.

4. Kosiński, W. On fuzzy number calculus. Int. J. Appl. Math. Comput. Sci. 2006, 16, 51-57.

5. Goetschel, R.; Voxman, W. Elementary fuzzy calculus. Fuzzy Sets Syst. 1986, 18, 31-43. [CrossRef]

6. Piasecki, K. Revision of the Kosiński's Theory of Ordered Fuzzy Numbers. Axioms 2018, 7, 16. [CrossRef]

7. Apiecionek, Ł. Fuzzy Observation of DDoS Attack. In Theory and Applications of Ordered Fuzzy Number; Prokopowicz, P., Czerniak, J., Mikołajewski, D., Apiecionek, Ł., Ślęzak, D., Eds.; Springer: Berlin, Germany, 2017; pp. 239-252.

8. Apiecionek, Ł. Fuzzy Control for Secure TCP Transfer. In Theory and Applications of Ordered Fuzzy Number; Prokopowicz, P., Czerniak, J., Mikołajewski, D., Apiecionek, Ł., Ślęzak, D., Eds.; Springer: Berlin, Germany, 2017; pp. 253-268.

9. Chwastyk, A.; Pisz, I. OFN Capital Budgeting Under Uncertainty and Risk. In Theory and Applications of Ordered Fuzzy Number; Prokopowicz, P., Czerniak, J., Mikołajewski, D., Apiecionek, Ł., Ślęzak, D., Eds.; Springer: Berlin, Germany, 2017; pp. 157-170.

10. Czerniak, J.M. OFN Ant Method Based on TSP Ant Colony Optimization. In Theory and Applications of Ordered Fuzzy Number; Prokopowicz, P., Czerniak, J., Mikołajewski, D., Apiecionek, Ł., Ślęzak, D., Eds.; Springer: Berlin, Germany, 2017; pp. 207-222.

11. Dobrosielski, W.T.; Czerniak, J.M.; Zarzycki, H.; Szczepański, J. Fuzzy Numbers Applied to a Heat Furnace Control. In Theory and Applications of Ordered Fuzzy Number; Prokopowicz, P., Czerniak, J., Mikołajewski, D., Apiecionek, Ł., Ślęzak, D., Eds.; Springer: Berlin, Germany, 2017; pp. 269-288.

12. Ewald, D.; Czerniak, J.M.; Paprzycki, M. A New OFN Bee Method as an Example of Fuzzy Observance Applied for ABC Optimization. In Theory and Applications of Ordered Fuzzy Number; Prokopowicz, P., Czerniak, J., Mikołajewski, D., Apiecionek, Ł., Ślęzak, D., Eds.; Springer: Berlin, Germany, 2017; pp. $223-238$.

13. Kacprzak, D. Input-Output Model Based on Ordered Fuzzy Numbers. In Theory and Applications of Ordered Fuzzy Number; Prokopowicz, P., Czerniak, J., Mikołajewski, D., Apiecionek, Ł., Ślȩzak, D., Eds.; Springer: Berlin, Germany, 2017; pp. 171-182.

14. Kacprzak, D. Objective weights based on ordered fuzzy numbers for fuzzy multiple criteria decision-making methods. Entropy 2017, 19, 373. [CrossRef]

15. Kacprzak, D.; Kosiński, W. Optimizing Firm Inventory Costs as a Fuzzy Problem. Stud. Logic Grammar Rhetor. 2014, 37, 89-105. [CrossRef]

16. Kacprzak, D.; Kosiński, W.; Kosiński, W.K. Financial Stock Data and Ordered Fuzzy Numbers. In Proceedings of the 12th International Conference on Artificial Intelligence and Soft Computing: (ICAISC 2013), Zakopane, Poland, 9-13 June 2013; pp. 259-270.

17. Łyczkowska-Hanćkowiak, A. Behavioural present value determined by ordered fuzzy number. SSRN Electr. J. 2017. [CrossRef]

18. Łyczkowska-Hanćkowiak, A. Oriented behavioural present value-Revised approach. In Methods and Models of Regional Development, Proceedings of the 9th International Scientific Conference: Analysis of International Relations 2018 (Summer Edition Conference), Katowice, Poland, 19-20 June 2018; Szkutnik, W., Sączewska-Piotrowska, A., Hadaś-Dyduch, M., Acedański, J., Eds.; Publishing House of the University of Economics in Katowice: Katowice, Poland, 2018; pp. 14-21.

19. Łyczkowska-Hanćkowiak, A.; Piasecki, K. The expected discount factor determined for present value given as ordered fuzzy number. In Methods and Models of Regional Development, Proceedings of the 9th International Scientific Conference Analysis of International Relations 2018 (Winter Edition Conference), Katowice, Poland, 12 January 2018; Szkutnik, W., Sączewska-Piotrowska, A., Hadaś-Dyduch, M., Acedański, J., Eds.; Publishing House of the University of Economics in Katowice: Katowice, Poland, 2018; pp. 69-75.

20. Łyczkowska-Hanćkowiak, A.; Piasecki, K. On representation of Japanese candlesticks by ordered fuzzy numbers. In Methods and Models of Regional Development, Proceedings of the 9th International Scientific Conference Analysis of International Relations 2018 (Winter Edition Conference), Katowice, Poland, 12 January 2018; Szkutnik, W., Sączewska-Piotrowska, A., Hadaś-Dyduch, M., Acedański, J., Eds.; Publishing House of the University of Economics in Katowice: Katowice, Poland, 2018; pp. 61-68. 
21. Łyczkowska-Hanćkowiak, A.; Piasecki, K. Two-assets portfolio with trapezoidal oriented fuzzy present values. In Proceedings of the 36th International Conference Mathematical Methods in Economics Conference,

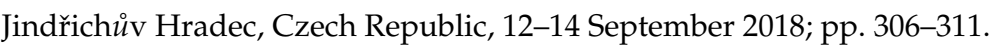

22. Łyczkowska-Hanćkowiak, A.; Piasecki, K. Present value of portfolio of assets with present values determined by trapezoidal ordered fuzzy numbers. Oper. Res. Decis. 2018, 28, 41-56.

23. Marszałek, A.; Burczyński, T. Ordered fuzzy candlesticks. In Theory and Applications of Ordered Fuzzy Number; Prokopowicz, P., Czerniak, J., Mikołajewski, D., Apiecionek, Ł., Ślęzak, D., Eds.; Springer: Berlin, Germany, 2017; pp. 183-194.

24. Piasecki, K. Expected return rate determined as oriented fuzzy number. In Proceedings of the 35th International Conference Mathematical Methods in Economics Conference, Hradec Králové, Czech Republic, 13-15 September 2017; pp. 561-565.

25. Piasecki, K.; Łyczkowska-Hanćkowiak, A. Japanese candle for financial portfolio. In Proceedings of the 36th International Conference Mathematical Methods in Economics Conference, Jindřich $u$ v Hradec, Czech Republic, 12-14 September 2018; pp. 444-449.

26. Piasecki, K.; Roszkowska, E. On application of ordered fuzzy numbers in ranking linguistically evaluated negotiation offers. Adv. Fuzzy Syst. 2018, in press.

27. Prokopowicz, P.; Mikołajewska, E.; Mikołajewski, D.; Kotlarz, P. Analysis of Temporospatial Gait Parameters. In Theory and Applications of Ordered Fuzzy Number; Prokopowicz, P., Czerniak, J., Mikołajewski, D., Apiecionek, Ł., Ślęzak, D., Eds.; Springer: Berlin, Germany, 2017; pp. 289-302.

28. Prokopowicz, P.; Mikołajewski, D. OFN-Based Brain Function Modeling. In Theory and Applications of Ordered Fuzzy Number; Prokopowicz, P., Czerniak, J., Mikołajewski, D., Apiecionek, Ł., Ślęzak, D., Eds.; Springer: Berlin, Germany, 2017; pp. 303-322.

29. Roszkowska, E.; Kacprzak, D. The fuzzy SAW and fuzzy TOPSIS procedures based on ordered fuzzy numbers. Inf. Sci. 2016, 369, 564-584. [CrossRef]

30. Rudnik, K.; Kacprzak, D. Fuzzy TOPSIS method with ordered fuzzy numbers for flow control in a manufacturing system. Appl. Soft Comput. 2017, 52, 1020-1041. [CrossRef]

31. Zarzycki, H.; Czerniak, J.M.; Dobrosielski, W.T. Detecting Nasdaq Composite Index Trends with OFNs. In Theory and Applications of Ordered Fuzzy Number; Prokopowicz, P., Czerniak, J., Mikołajewski, D., Apiecionek, Ł., Ślęzak, D., Eds.; Springer: Berlin, Germany, 2017; pp. 195-206.

32. Prokopowicz, P.; Czerniak, J.; Mikołajewski, D.; Apiecionek, Ł.; Ślęzak, D. Theory and Applications of Ordered Fuzzy Number; Springer: Berlin, Germany, 2017.

33. Klir, G.J. Developments in uncertainty-based information. Adv. Comput. 1993, 36, 255-332.

34. De Luca, A.; Termini, S. Entropy and energy measures of fuzzy sets. In Advances in Fuzzy Set Theory and Applications; Gupta, M.M., Ragade, R.K., Yager, R.R., Eds.; Elsevier: Amsterdam, The Netherlands, 1979; pp. 321-338.

35. De Luca, A.; Termini, S. A definition of a non-probabilistic entropy in the settings of fuzzy set theory. Inf. Control 1972, 20, 301-312. [CrossRef]

36. Piasecki, K. Some remarks on axiomatic definition of entropy measure. J. Intell. Fuzzy Syst. 2017, 33, 1945-1952. [CrossRef]

37. Dubois, D.; Prade, H. Operations on fuzzy numbers. Int. J. Syst. Sci. 1978, 9, 613-626. [CrossRef]

38. Dubois, D.; Prade, H. Fuzzy real algebra: some results. Fuzzy Sets Syst. 1979, 2, 327-348. [CrossRef]

39. Piasecki, K. Behavioural Present Value. SSRN Electr. J. 2011. [CrossRef]

40. Piasecki, K.; Siwek, J. Behavioural Present Value Defined as Fuzzy Number a new Approach. Folia Oecon. Stetin. 2015, 15, 27-41. [CrossRef]

41. Zadeh, L.A. Fuzzy sets. Inf. Control 1965, 8, 338-353. [CrossRef]

42. Zadeh, L.A. Computing with Words in Information/Intelligent Systems 1: Foundations; Physica-Verlag: Heidelberg, Germany, 1999.

43. Zadeh, L.A. Computing with Words in Information/Intelligent Systems 2: Applications; Physica-Verlag: Heidelberg, Germany, 1999.

44. Khalili, S. Fuzzy measures and mappings. J. Math. Anal. Appl. 1979, 68, 92-99. [CrossRef]

45. Kosko, B. Fuzzy entropy and conditioning. Inf. Sci. 1986, 40, 165-174. [CrossRef]

46. Shores, T.S. Applied Linear Algebra and Matrix Analysis; Springer: Berlin, Germany, 2007. 
47. Garcia-Lapresta, J.L.; Piggins, A. Voting on how to vote. In Soft Computing in Humanities and Social Sciences; Seising, R., Sanz, V., Eds.; Springer: Berlin, Germany, 2012; Volume 273, pp. 301-321.

48. Alcantud, J.C.R.; Biondo, A.E.; Giarlotta, A. Fuzzy politics I: The genesis of parties. Fuzzy Sets Syst. 2018, 349, 71-98. [CrossRef]

(․) (1) (C) 2018 by the authors. Licensee MDPI, Basel, Switzerland. This article is an open access article distributed under the terms and conditions of the Creative Commons Attribution (CC BY) license (http:/ / creativecommons.org/licenses/by/4.0/). 